\title{
Full Covered Path Planning Algorithm for Portable Back Massage Robot
}

Wendong Wang*, Jinzhe Li, Zhicheng Xu, Xiaoqing Yuan and Yikai Shi

School of Mechanical Engineering, Northwestern Polytechnical University, Xi'an, Shaanxi, China

\begin{abstract}
The massage coverage and the massage path have significant influence on the massage effect of massage robot. Analyzing the traditional Chinese massage operation and the demand for path planning, an efficient full covered improved path planning algorithm which based on a portable back massage robot was put forward. The model of an improved back and forth path algorithm and an efficient full covered path algorithm were established and the simulation were implemented in Matlab. The simulation results show that the improved algorithm is suitable for back massage, and it ensures that the massage robot traverses the entire back area with improved massage coverage. Meanwhile, the experimental environment was built to test the effect of the proposed algorithm. A series of problems appeared during experiments were solved with repeated tests; the massage robot eventually achieved the desired massage effect and planned path.
\end{abstract}

Keywords: Massage robot; Path planning; Simulation experiment; Coverage

\section{Introduction}

Massage equipment can effectively alleviate the shortage of masseur/ masseuse, help to promote blood circulation, eliminate muscle fatigue and improve the quality of life for the elderly and bone/muscle disease people. Numbers of research institutes carried out research work of massage robots to improve massage effect [1-3]. Our group has paid great effort to design a novel wheel massage robot with the features of portable structure, multi-function and high intelligence [4].

Currently, the complete coverage path planning of mobile robot has been widely studied, such as stochastic method, spiral covering algorithm (ISC), complete coverage based on the template and Boustrophedon reciprocating forward method, etc. [5-8]. Stochastic method is to allow the robot to select a random direction, and steer to move forward when encountering an obstacle. The algorithm is simple, but it cannot ensure that the area is completely covered. The basic idea of the spiral algorithm is to make the robot move forward in a certain direction (clockwise or counterclockwise), if the front of the area is not covered and then the robot moves on, conversely, if the front of the area is covered or has an obstacle, then the robot turns right 90 , until it covers the entire area. The complete coverage path planning algorithm based on the template requests the environment map known, and the different terrain needs different templates. This method can achieve complete coverage, but the price is to build the environmental map in advance. Boustrophedon reciprocating forward method will exist uncovered area, which is caused by obstructions, and the more obstacles, the more uncovered areas.

However, the research object of this paper is different from the other service robot with the functions and work environment. The existing path planning algorithms can not directly used for the presented object. Therefore, this paper proposed a high-coverage path planning algorithm to improve the level of intelligence and completed the simulation and experimental tests of the path planning.

\section{Requirements of Path Planning}

This paper focused on the portable back massage robot which was developed in our group, the $3 \mathrm{D}$ model and prototype are shown in Figure 1. The goal of the design is to relax the user by working on the whole back area. There are two massage paths based on the wheel massage robot, namely tapping path and rolling/kneading path. Among them, tapping path is the path of the tapping head of electromagnetic induction; rolling/kneading path is the motion path of the two drive wheels.

To get better massage effect, the requirements for path planning of massage robot are given as follows:

(1) Massage path must cover the entire massage area with high coverage;

(2) Be able to avoid all the obstacles and the edge of the human back;

(3) Walking path is composed of simple trajectory (straight line, arc);

(4) Duplicate path is allowed.

\section{Analysis of the Algorithm}

The flow chart of the high full covered algorithm is indicated in

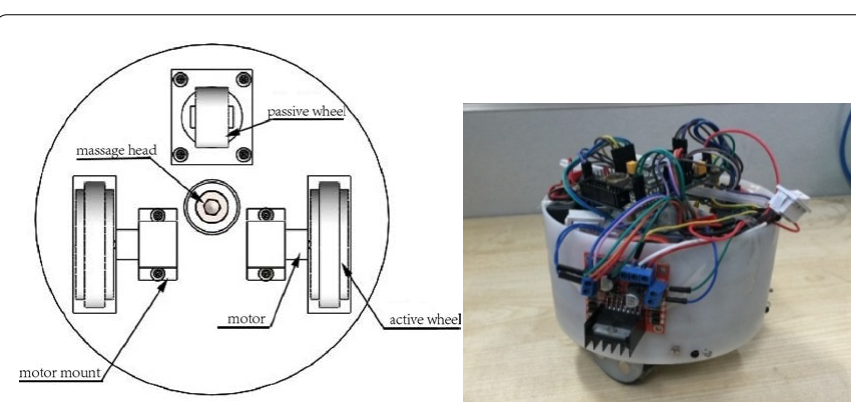

Figure 1: 3D model and prototype of massage robot: $(A) 3 D$ model; $(B)$ Prototype of massage robot.

*Corresponding author: Wendong Wang, School of Mechanical Engineering Northwestern Polytechnical University, X'an, Shaanxi, China, Tel: +8602988494893; E-mail: wdwang@nwpu.edu.cn

Received July 10, 2017; Accepted August 04, 2017; Published August 16, 2017

Citation: Wang W, Li J, Xu Z, Yuan X, Shi Y (2017) Full Covered Path Planning Algorithm for Portable Back Massage Robot. Adv Robot Autom 6: 167. doi: 10.4172/2168-9695.1000167

Copyright: $\odot 2017$ Wang W, et al. This is an open-access article distributed under the terms of the Creative Commons Attribution License, which permits unrestricted use, distribution, and reproduction in any medium, provided the original author and source are credited. 
Citation: Wang W, Li J, Xu Z, Yuan X, Shi Y (2017) Full Covered Path Planning Algorithm for Portable Back Massage Robot. Adv Robot Autom 6: 167. doi: $10.4172 / 2168-9695.1000167$

Page 2 of 5

Figure 2. The reciprocating algorithm is taken when the program is started. The backward method is used when there is obstacle or area edge. The BFS (Breadth-First Search) algorithm would be used to search the nearest uncovered area if the surrounding has been covered. The search would not be stopped until the whole area has been covered.

The improved reciprocating algorithm is proposed based on the traditional reciprocating algorithm by re-covering, the trajectory scheme is described in Figure 3. From the trajectory, it can be seen that the robot walk along $\mathrm{x}$ axis, it would move along $\mathrm{y}$ axis as far as the length of the robot body and then it walks to the opposite of $\mathrm{x}$ axis. It would decide if there is edge or obstacle in the front of the robot body before moving along y axis. If yes, it has to take the backward order. After the first coverage, the robot would start the second coverage when it moves a half length of the body. Then, the surrounding uncovered area will be searched based on BFS algorithm. The directional grid is

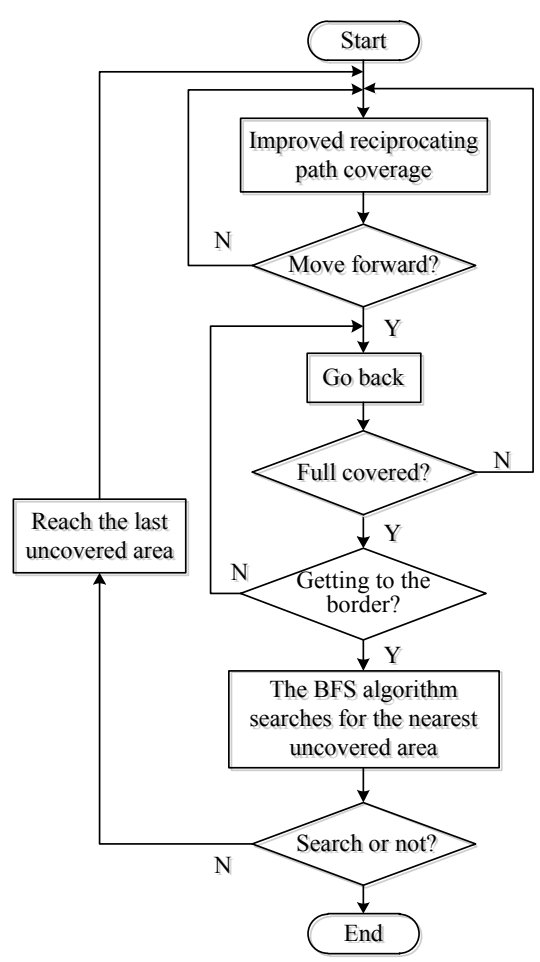

Figure 2: Effective full covered path planning algorithm flow chart.

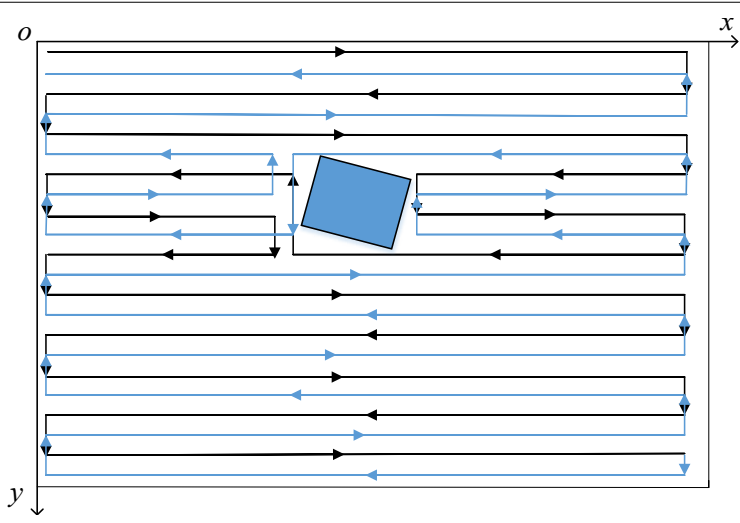

Figure 3: The improved back and forth trajectory. designed as Figure 4. The black square is the current location of the robot, and the arrow shows the searching direction. For the directional grid, the length of the square is defined as 1.

\section{Modeling and Simulation}

\section{Control system modeling}

This paper obtained the 3D model to build the simulation object in Solid works, and then established the control system model of wheel massage robot based on Simulink, which is indicated in Figure 5. The control system model includes control subsystem of the motor, path algorithm control subsystem, parameter module of the massage robot and virtual reality interface module. Control signal is directly outputted to a virtual operating environment by virtual reality (VR) sink block, the operation of the massage robot could be visual through a browser window.

\section{Analysis of the simulation}

Three assumptions were proposed for the control system program to implement simulation.

(1) Assuming that the starting point is close to the edge of human back;

(2) Assuming that both wheels and massage head occupy a grid, then the whole robot occupies nine grids;

(3) Assuming that the four beneath sensors can exactly detect the path edge, and the two front sensors can detect the obstacles on the head and waist.

From the above design and analysis, it is known that the walking area of the massage robot is not the massage area. Thus, this paper chose

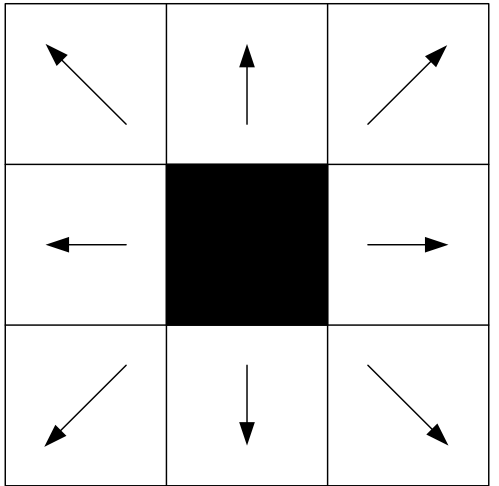

Figure 4: 8 Directional grid

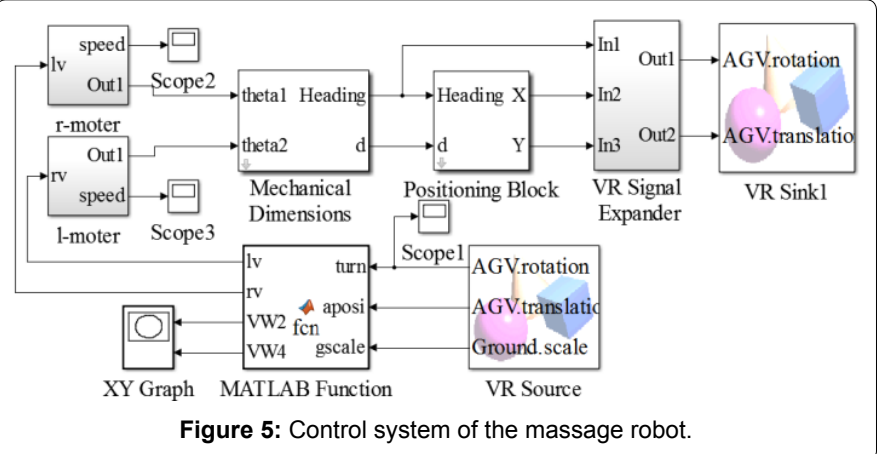


Citation: Wang W, Li J, Xu Z, Yuan X, Shi Y (2017) Full Covered Path Planning Algorithm for Portable Back Massage Robot. Adv Robot Autom 6: 167. doi: $10.4172 / 2168-9695.1000167$

Page 3 of 5

the tapping path as the study object, and simulated the massage path with traditional and improved reciprocating path planning algorithm. Coverage rate is an important indicator to measure the effectiveness of the complete coverage path planning algorithm, the coverage rate was calculated as: Coverage $=$ Haven covered grid/All grids

The simulation results of the proposed algorithm before and after improving of tapping path were shown in Figure 6. From the results, it can be calculated that the coverage rate is only $35.27 \%$ using basic reciprocating path planning algorithm while it reaches up to $60.27 \%$ by the improved reciprocating path planning algorithm. It indicates that the improved reciprocating path planning algorithm proposed in this paper can greatly improve coverage rate, and then the massage effect gets improved. For rolling/kneading path, the corresponding results are shown in Figure 7. It can be seen from the figure, the calculated coverage rate is only $58.82 \%$ using basic reciprocating path planning algorithm as shown in Figure $7 \mathrm{~A}$, it is improved to $84.93 \%$ using the improved reciprocating path planning algorithm as shown in Figure 7B.

From the simulation results, the improved path planning algorithm greatly improved the coverage. However, the structure and the working area of the massage robot causes the improvement is restricted as the robot body can not be taken as a particle compared to the working area. Therefore, this paper proposed a high efficiency full covered path planning algorithm based on grid map.
Taking an example of tapping path, the simulation result is shown in Figure 8 by improved reciprocating path planning algorithm, the white area is the uncovered area, and the calculated coverage is $80 \%$. Combining the improved reciprocating algorithm and the BFS algorithm, the simulation result is given in Figure 9, it indicates that the coverage reaches up to $100 \%$. With the above results and analysis, it can be concluded that the proposed high efficiency full covered path planning algorithm is suitable for limited and regular environment, and the specific environment information is not required in advance.

\section{Experimental Tests and Analysis}

To verify the feasibility of the presented algorithm and test the accuracy of the motion control system, an experimental setup was constructed with the previous wheeled robot (Figure 10), and the results were recorded.

Furthermore, to test the performance of the path planning, the experimental enviroment was built as shown in Figure 11. In the planned area, there are two walls to simulate obstacle and the desk edge to simulate the back edge. This setting was used to test if the massage robot could walk along the planned route and avoid the detected edge. Then, the full coverage effect can be calculated.

For the preliminary test, it was carried out 30 times in total, 18 of them succeeded and 12 of them failed. The success was defined as
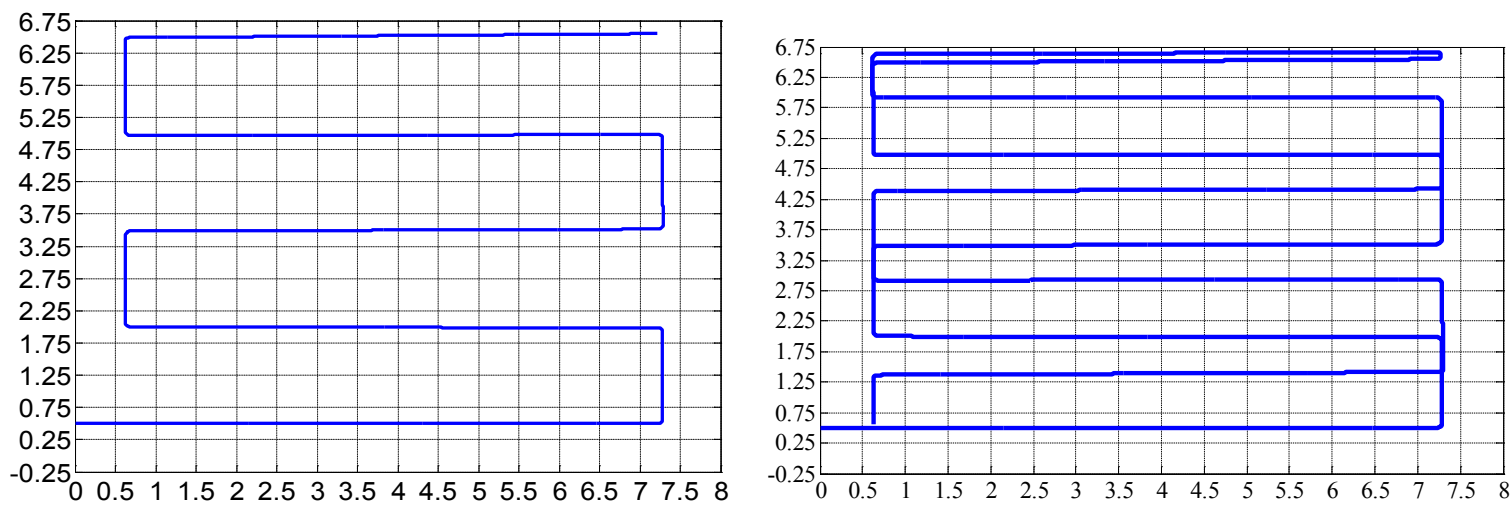

Figure 6: The tapping path algorithm before and after improvement: (A) The algorithm before improvement; (B) The algorithm after improvement
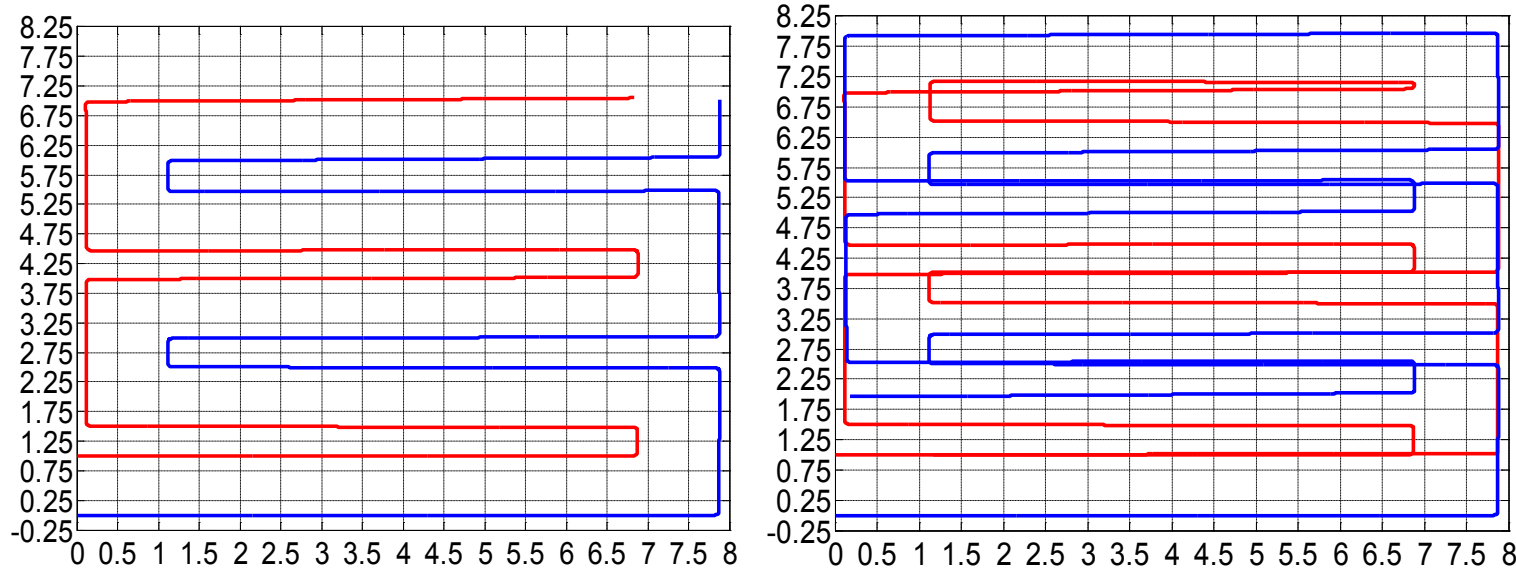

Figure 7: Comparison before and after improving of rolling/kneading path: (A) Algorithm before improving; (B) Algorithm after improving . 
Citation: Wang W, Li J, Xu Z, Yuan X, Shi Y (2017) Full Covered Path Planning Algorithm for Portable Back Massage Robot. Adv Robot Autom 6: 167. doi: $10.4172 / 2168-9695.1000167$

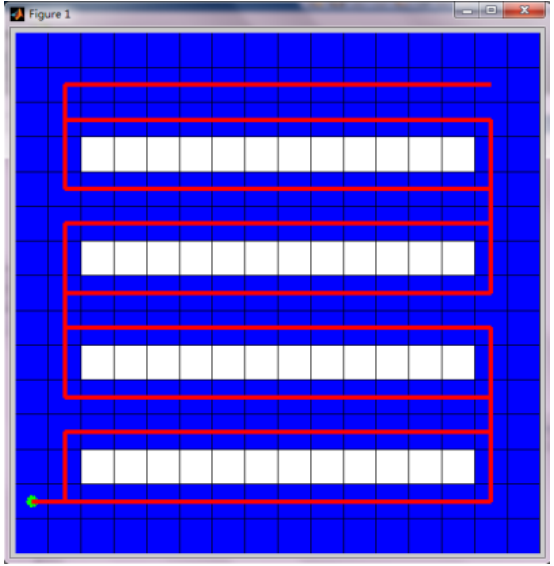

Figure 8: Improved algorithm with running a circle.

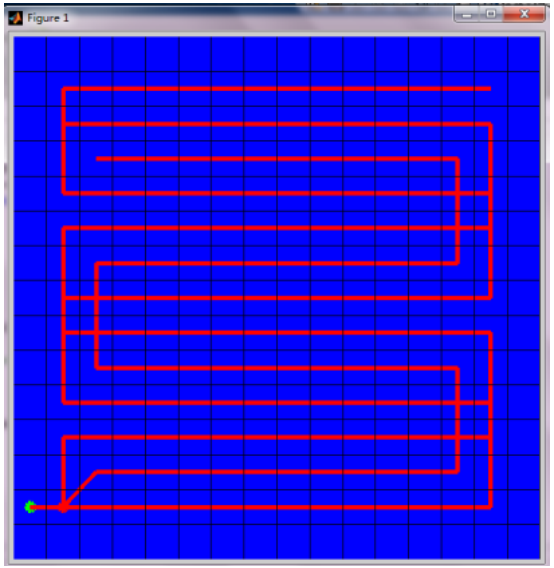

Figure 9: Using the BFS search algorithm.

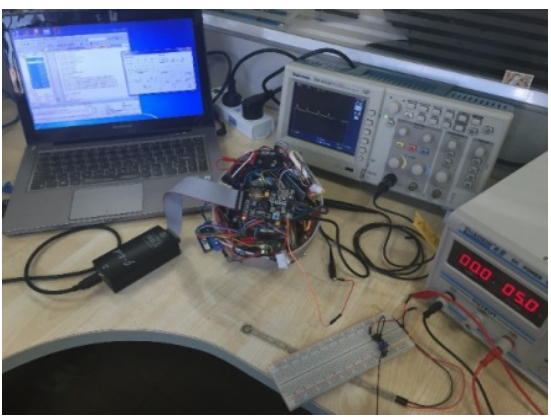

Figure 10: Experimental setup of the system

when the robot walked along the planned route and does not lose the direction. Some problems were recorded, for example,

(1) The robot did not achieved expected $90^{\circ}$ when turned around;

(2) There were deviation when it walks in line;

(3) There were uncovered area on the border.

The possible reasons of failed route were discussed, such as,

(1) There was deformation on the axes of the wheels, and the robot could not walk on the same plane;
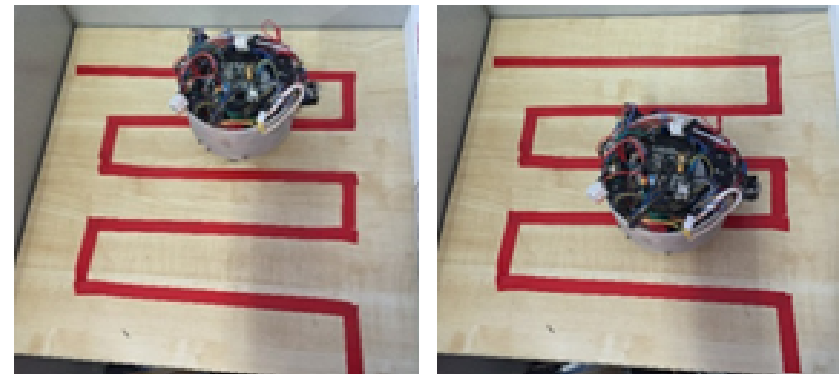

Figure 11: Experimental environment of path planning.

(2) The distance of the sensors was not reasonably;

(3) Blind angle was not considered during programming.

Some improvements were made with the above analysis for the problems and possible reasons. Another 30 tests were implemented in the same environment, 28 of them succeeded and 2 of them failed. The success rate reached up to $93.3 \%$ from $60 \%$, it is relatively satisfied. Meanwhile, it proves the aforementioned design of the experimental setup is reasonable, and the proposed high full covered path planning algorithm improved the coverage.

\section{Conclusion}

This paper proposed a high full covered path planning algorithm and established the control system model by using Matlab/Simulink platform. The simulation of traditional and improved reciprocating path planning algorithm were performed with the above model. The simulation results show that the proposed algorithm can be used in limited massage area, and there is no need to build the environment model in advance. The motion control and path planning test environment was built based on the previous wheeled massage robot prototype. With the analysis and discussion for the appeared problems, the program and design of the robot were optimized to improve the performance of the control system. By comparing the simulation and experimental results, it can be concluded that the proposed algorithm can effectively improve the coverage for massage robot, and the massage effect also gets improved.

\section{Acknowledgments}

This work is supported by National Natural Science Foundation of China (Grant No. 51605385), Natural Science Foundation of Shaanxi Province (Grant No. 2017JQ5026) and Fundamental Research Funds for the Central Universities (Grant No. 3102017zy008).

\section{References}

1. Wendong W, Qinqin J, Zhicheng X, Lijing H, Yikai S (2016) Structure optimization and force control of electromagnetic induction-based massage robot hand. Machinery \& Electronics 34: 71-75+80.

2. Jamwal PK, Hussain S, Ghayesh MH, Rogozina SV (2016) Impedance Control of an Intrinsically Compliant Parallel Ankle Rehabilitation Robot. leee Transactions on Industrial Electronics 63: 3638-3647.

3. Fazeli MS, Pourrahmat MM, Liu M, Guan L, Collet JP (2016) The Effect of Head Massage on the Regulation of the Cardiac Autonomic Nervous System: A Pilot Randomized Crossover Trial. J Altern Complement Med 22: 75-80.

4. Wendong W, Lijing H, Qinqin J, Peng Z, Yikai S (2016) Path Planning Algorithm of a Novel Massage Robot. In: 2016 9th International Congress on Image and Signal Processing, BioMedical Engineering and Informatics(CISP-BMEI 2016). Datong, China.

5. Batsaikhan D, Janchiv A, Lee SG (2013) Sensor-Based Incremental Boustrophedon Decomposition for Coverage Path Planning of a Mobile Robot In: 12th International Conference of Intelligent Autonomous Systems 193 621-628. 
Citation: Wang W, Li J, Xu Z, Yuan X, Shi Y (2017) Full Covered Path Planning Algorithm for Portable Back Massage Robot. Adv Robot Autom 6: 167. doi: 10.4172/2168-9695.1000167

Page 5 of 5

6. Viet HH, Dang VH, Choi S, Chung T (2015) BoB: an online coverage approach for multi-robot systems. Applied Intelligence 42: 157-173.

7. Liu SH, Xia J, Sun XM (2011) An Efficient Complete Coverage Path Planning
Algorithm of Known Environment. Northeast Normal University (Natura Science Edition) 04: 39-43.

8. Zhao HN, Liu SH, Wu FZ (2011) Complete Coverage Planning Algorithm Based on The Binary Search of Cattle Farming Formula. Computer Engineering and Applications 23: 51-53+60. 